\title{
An improved analytical method for triple stub matching (Stubs in Parallel)
}

\author{
Slobodan Babic ${ }^{1, *}$ and Cevdet Akyel ${ }^{2}$ \\ ${ }^{1}$ Independent Researcher, 53 Berlioz, 101, H3E 1N2, Montréal, Canada. \\ 2 Département de Génie Électrique, École Polytechnique, C.P. 6079 Succ. Centre-Ville, QC H3C 3A7, Montréal, Canada.
}

Global Journal of Engineering and Technology Advances, 2021, 07(03), 157-178

Publication history: Received on 15 May 2021; revised on 18 June 2021; accepted on 21 June 2021

Article DOI: https://doi.org/10.30574/gjeta.2021.7.3.0089

\begin{abstract}
In this paper we give the new improved analytical method of triple stub tuner for matching the load impedances to provide the maximum power transfer between a generator and a load. The stubs are connected in parallel with the line at the appropriate distances from the load. The characteristic impedances of the transmission line and the stabs are different. The paper points on the determination of the length for the first stub near the load. The limit lengths for the first and the second stub are found for which the matching is possible. The length of the third stub is directly obtained from the matching conditions. Even though there is not the unique solution for the triple stub matching we also shoved the existence of unique solutions under some conditions. The special cases are also treated. The results of this method are verified by those obtained using the Smith chart and there are in exceptionally good agreement. Even though there are not many papers on this subject this work could be useful for engineers, and physicist which work in this domain.
\end{abstract}

Keywords: Impedance matching; Transmission line; Short or open stub; Triple stub in parallel

\section{Introduction}

In electronics, microwave and $R F$ engineering, the impedance matching is the practice of designing the input impedance of an electrical load or the output impedance of its corresponding signal source to maximize the power transfer or minimize signal reflection from the load. In general, for the matching, the stubs are widely used to match any complex load to a transmission line. They consist of shorted or opened segments of the line, connected in parallel or in series with the line at the appropriate distances from the load and with their appropriate lengths. In the case of the single stub the distance from the load and its length are required for given the load impendence as well as the characteristic impedances of the line and the stub. Single stubs can match any load impendence to a transmission line, but the problem is a variable distance between the stab and the load. To overcome the drawbacks of the single-stub matching technique, the double-stub matching technique is employed. This is way the double stabs are preferable because they are inserted at predetermined locations. Thus, for given distance between stubs and the given position from the first stub and the load, the stub lengths are required. A disadvantage of double stubs is that they cannot match all loads. To overcome this problem, triple stubs are used, [1]-[11]. With them all loads can be matched. The distances between the second and the third stub, the second and the first stub are given as well as the distance between the first stub and the load. The stub lengths are required. These stubs can be connected in parallel or in series with the line as in the case of the double or single stubs. Even though there is no unique solution for triple stub matching we think that the presented method could be a useful contribution on this subject. Also, we found the unique solution in the triple stub matching under some conditions. As it was mentioned we will work with the different impedances everywhere. The short-circuited stubs are preferred to open circuited stubs because the latter radiate some energy at high frequencies. Moreover, all combinations either for short stubs or for open stubs can be treated with this approach.

\footnotetext{
${ }^{*}$ Corresponding author: Slobodan Babic

Independent Researcher, 53 Berlioz, 101, H3E 1N2, Montréal, Canada.
}

Copyright $(2021$ Author(s) retain the copyright of this article. This article is published under the terms of the Creative Commons Attribution Liscense 4.0. 


\section{Analytical Method}

The load impedance $Z_{L}=A+j B,(A, B \in R)$ of the transmission line with the characteristic impendence $Z_{0}$ and without losses, is to be matched by a triple-stub tuner connected in parallel (Fig. 1). The first stub is distanced from the load by $d_{1}$. The distance between the first and the second stub is $d_{2}$ and the distance between the second and the third stub is $d_{3}$. Their characteristic impendences are respectively $Z_{S 1}=k_{1} Z_{0}, Z_{S 2}=k_{2} Z_{0}$ and $Z_{S 3}=k_{3} Z_{0}$. the lengths $l_{1}$, $l_{2}$ and $l_{3}$ are required to find. In this paper all impedances or admittances are normalized.

The normalized load admittance is,

$$
y_{L N}=\frac{1}{y_{L N}}=p_{L}+j q_{L}
$$

where

$$
p_{L}=\frac{A}{Y_{0}\left(A^{2}+B^{2}\right)}, q_{L}=\frac{-B}{Y_{0}\left(A^{2}+B^{2}\right)}
$$

Let us calculate the normalized admittance at points $A$,

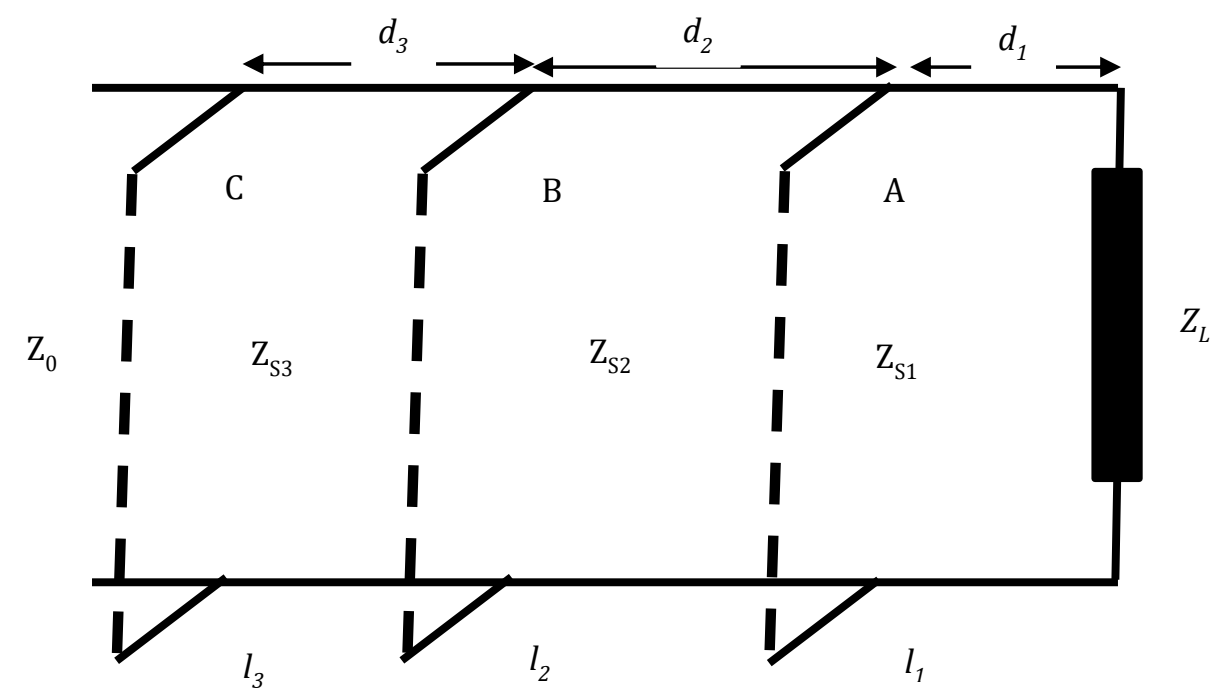

Figure 1 Triple short stubs in parallel (S/S/S; S/S/O; O/S/S; O/S/O/ S/O/S; S/O/O; O/O/S; O/O/O)

$$
y_{A}=y_{\left(d_{1}\right)}=\frac{y_{L N}+j \tan \left(\beta d_{1}\right)}{1+j \tan \left(\beta d_{1}\right) y_{L N}}=\frac{y_{L N}+j a}{1+j a y_{L N}}=g_{L A}+j b_{L A}
$$

where

$$
\begin{gathered}
a=\tan \left(\beta d_{1}\right), a \in R \\
g_{L A}=\frac{p_{L}\left(1+a^{2}\right)}{\left(1-a q_{L}\right)^{2}+a^{2} p_{L}^{2}} \\
b_{L A}=\frac{a\left(1-p_{L}^{2}-q_{L}^{2}\right)+q_{L}\left(1-a^{2}\right)}{\left(1-a q_{L}\right)^{2}+a^{2} p_{L}^{2}}
\end{gathered}
$$

The total admittance at points $A$ is, 


$$
y_{\text {TotalA }}=y_{A}+j y_{S 1}=g_{L A}+j\left(b_{L A}+B_{S 1}\right)=g_{L A}+j B_{1}
$$

where

$$
y_{S 1}=j B_{S 1}
$$

$$
\begin{aligned}
& B_{1}=b_{L A}+B_{S 1} \\
& B_{S 1}=-\frac{1}{z_{S 1}}=-\frac{1}{k_{1} b}, z_{S 1}=k_{1} \tan \left(\beta l_{1}\right)=k_{1} b,\left(\frac{S}{C}\right) \\
& B_{S 1}=\frac{1}{z_{S 1}}=\frac{b}{k_{1}}, \quad z_{S 1}=\frac{\tan \left(\beta l_{1}\right)}{k_{1}}=\frac{b}{k_{1}},\left(\frac{O}{C}\right) \\
& b=\tan \left(\beta l_{1}\right),
\end{aligned}
$$

The next step is the determination of the admittance at point B.

Now, the admittance at the point $B$ is,

$$
y_{B}=\frac{y_{\text {TotalA }}+j \tan \left(\beta d_{2}\right)}{1+j y_{\text {TotalA }} \tan \left(\beta d_{2}\right)}=\frac{y_{\text {TotalA }}+j m}{1+j y_{\text {TotalA }} m}=g_{L B}+j b_{L B}
$$

where

$$
\begin{aligned}
& \quad m=\tan \left(\beta d_{2}\right) \\
& g_{L B}=\frac{g_{L A}\left(1+m^{2}\right)}{\left(1-m B_{1}\right)^{2}+m^{2} g_{L A}^{2}} \\
& b_{L B}=\frac{m\left(1-g_{L A}^{2}-B_{1}^{2}\right)+B_{1}\left(1-m^{2}\right)}{\left(1-m B_{1}\right)^{2}+m^{2} g_{L A}^{2}}
\end{aligned}
$$

The total admittance at the point B is,

$$
y_{\text {TotalB }}=y_{B}+j y_{S 2}=g_{L B}+j\left(b_{L B}+B_{S 2}\right)=g_{L B}+j B_{2}
$$

with

$$
B_{2}=b_{L B}+B_{S 2}
$$

where

$$
\begin{gathered}
B_{S 2}=-\frac{1}{z_{S 2}}=-\frac{1}{k_{2} n}, z_{S 2}=k_{2} \tan \left(\beta l_{2}\right)=k_{2} n,\left(\frac{S}{C}\right) \\
B_{S 2}=\frac{1}{z_{S 2}}=\frac{n}{k_{2}}, \quad z_{S 2}=\frac{\tan \left(\beta l_{2}\right)}{k_{2}}=\frac{n}{k_{2}},\left(\frac{O}{C}\right)
\end{gathered}
$$

The admittance at the point $\mathrm{C}$ is,

$$
y_{C}=\frac{y_{\text {TotalB }}+j \tan \left(\beta d_{3}\right)}{1+j y_{\text {TotalB }} \tan \left(\beta d_{3}\right)}=\frac{y_{\text {TotalB }}+j p}{1+j y_{\text {TotalB }} p}=g_{L C}+j b_{L C}
$$

with

$$
p=\tan \left(\beta d_{3}\right)
$$




$$
\begin{aligned}
g_{L C} & =\frac{g_{L B}\left(1+p^{2}\right)}{\left(1-p B_{2}\right)^{2}+p^{2} g_{L B}^{2}} \\
b_{L C} & =\frac{p\left(1-p_{L}^{2}-B_{2}^{2}\right)+B_{2}\left(1-p^{2}\right)}{\left(1-p B_{2}\right)^{2}+p^{2} g_{L B}^{2}}
\end{aligned}
$$

The total admittance at the point $\mathrm{C}$ is,

$$
y_{\text {TotalC }}=y_{C}+j y_{S 3}=g_{L C}+j\left(b_{L C}+B_{S 3}\right)=g_{L C}+j B_{3}
$$

with

$$
B_{3}=b_{L C}+B_{S 3}
$$

The condition of the matching at the point $\mathrm{C}$ is,

$y_{\text {TotalC }}=1$

which gives,

$g_{L C}=1$ or

$$
\frac{g_{L B}\left(1+p^{2}\right)}{\left(1-p B_{2}\right)^{2}+p^{2} g_{L B}^{2}}=1
$$

and

$b_{L C}+B_{S 3}=0$, or

$$
B_{S 3}=-\frac{p\left(1-g_{L B}^{2}-B_{2}^{2}\right)+B_{2}\left(1-p^{2}\right)}{\left(1-p B_{2}\right)^{2}+p^{2} g_{L B}^{2}}
$$

where,

$$
\begin{aligned}
& B_{S 3}=-\frac{1}{z_{S 3}}=-\frac{1}{k_{3} q}, z_{S 3}=k_{3} \tan \left(\beta l_{3}\right)=k_{3} q,\left(\frac{S}{C}\right) \\
& B_{S 3}=\frac{1}{z_{S 3}}=\frac{q}{k_{3}}, \quad z_{S 2}=\frac{\tan \left(\beta l_{3}\right)}{k_{3}}=\frac{q}{k_{3}},\left(\frac{O}{C}\right)
\end{aligned}
$$

The next step is determination of all stub lengths.

From (19) we obtain,

$$
\left(B_{2}-\frac{1}{p}\right)^{2}+g_{L B}^{2}=g_{L B}^{2} \frac{g_{(p)}}{g_{L B}}
$$

whose solutions fir $B_{2}$ are,

$$
B_{2}^{(1,2)}=\frac{1}{p} \pm g_{L B} \sqrt{\frac{g_{(p)}}{g_{L B}}-1}
$$

where

$$
g_{(p)}=\frac{1+p^{2}}{p^{2}}
$$


Equation (23) can have the real solutions if,

$$
\frac{g_{(p)}}{g_{L B}}-1 \geq 0
$$

or,

$$
g_{L B} \leq g_{(p)}=\frac{1+p^{2}}{p^{2}}
$$

Also, equation (23) can be written in the form,

$$
g_{L B}^{2}-g_{L B} g_{(p)}+\left(B_{2}-\frac{1}{p}\right)^{2}=0
$$

whose solutions are,

$$
g_{L B(1,2)}=\frac{g_{(p)} \pm \sqrt{g_{(p)}^{2}-4\left(B_{2}-\frac{1}{p}\right)^{2}}}{2}
$$

The following condition must be satisfied,

$$
g_{(p)}^{2}-4\left(B_{2}-\frac{1}{p}\right)^{2} \geq 0
$$

which gives,

$$
-\frac{(p-1)^{2}}{2 p^{2}} \leq B_{2} \leq \frac{(p+1)^{2}}{2 p^{2}}
$$

or

$$
-g_{L B(i)}-\frac{(p-1)^{2}}{2 p^{2}} \leq B_{S 2}^{(i)} \leq-g_{L B(i)}+\frac{(p+1)^{2}}{2 p^{2}}
$$

This condition is important because it gives the limits for $B_{2}$ or the susceptance for the second stub. Combining (7) and (26) we obtain,

$$
g_{L B}=\frac{g_{L A}\left(1+m^{2}\right)}{\left(1-m B_{1}\right)^{2}+m^{2} g_{L A}^{2}} \leq g_{(p)}
$$

or

$$
\frac{g_{L A} g_{(m)}}{\left(B_{1}-\frac{1}{m}\right)^{2}+g_{L A}^{2}} \leq g_{(p)}
$$

where

$$
g_{(m)}=\frac{1+m^{2}}{m^{2}}
$$

Let us introduce the constant $Q$ as, 


$$
Q=\frac{g_{(m)}}{g_{L A} g_{(p)}}=\frac{\left(1+m^{2}\right) p^{2}}{m^{2}\left(1+p^{2}\right) g_{L A}}>0, \forall m, p, g_{L A}
$$

The expression (30) can be written in the following form,

$$
\frac{g_{L A}^{2} Q}{\left(B_{1}-\frac{1}{m}\right)^{2}+g_{L A}^{2}} \leq 1
$$

This inequality presents the condition for finding the length of the first stub. Introducing the parameter $t \geq 1$, (33) can be written as,

$$
\frac{g_{L A}^{2} Q}{\left(B_{1}-\frac{1}{m}\right)^{2}+g_{L A}^{2}}=\frac{1}{t} \leq 1
$$

Let us take the limit of (34),

$$
\left(B_{1}-\frac{1}{m}\right)^{2}+g_{L A}^{2}=g_{L A}^{2} Q t
$$

or

$$
\left(B_{1}-\frac{1}{m}\right)^{2}=g_{L A}^{2}(Q t-1)
$$

I) $0<Q<1, t \geq 1$

I.1) If $1 \leq t \leq 1 / Q=g_{L A} \frac{g_{(p)}}{g_{(m)}}=t_{\max }$

$D=Q t-1=0$

that gives,

$$
\left(B_{1}-\frac{1}{m}\right)^{2}=0
$$

$$
B_{1}=\frac{1}{m}
$$

Equation (37) gives the susceptance for the first stub,

$$
B_{S 1}=-b_{L A}+\frac{1}{m}
$$

One can see that $B_{S 1}$ does not depend on ' $t$ '. Moreover, the susceptance for the second and the third stubs do not depend on ' $t$ ' by their definition. It is important because the unique triple stub matching is achieved for $1 \leq t \leq t_{\min }$ and $0<$ $Q<1$.

Thus, there is the unique solution (37) or (38) for the triple stub matching with (36).

I.2) If $0<Q<1, t>1 / Q$

$$
D=Q t-1>0
$$

that gives 


$$
B_{1}=\frac{1}{m}+g_{L A} \sqrt{Q t-1} \text { and } B_{1}=\frac{1}{m}-g_{L A} \sqrt{Q t-1}
$$

or

$B_{S 1}^{(1,2)}=-b_{L A}+\frac{1}{m} \pm g_{L A} \sqrt{Q t-1}$

with the condition,

$$
g_{L A} \leq \frac{\left(1+m^{2}\right) p^{2}}{m^{2}\left(1+p^{2}\right)} t
$$

II) $Q \geq 1, \quad t \geq 1$

For these values, this condition $Q t-1 \geq 0$ is satisfied automatically and the susceptances $B_{1}^{(1,2)}$ or $B_{S 1}^{(1,2)}$ for the first stub are determined by (39) and (40) with condition (41) for $g_{L A}$,

Equations (39) gives the limits where the matching is possible for the first stub.

$$
B_{1} \geq \frac{1}{m}+g_{L A} \sqrt{Q t-1} \text { and } B_{1} \leq \frac{1}{m}-g_{L A} \sqrt{Q t-1}
$$

From the previous analysis one can conclude that there is not the unique solution for $A$ ) If $0<Q<1, t>1 / Q$ and B) and $Q \geq 1, t \geq 1$. It could be an optimization problem. This is problem of the three variables where the lengths of the three stubs could be chosen to optimize the bandwidth of the matching.

The corresponding lengths for the first stub can be obtained for:

\subsection{Short circuit (stub)}

$$
B_{S 1}^{(1,2,3,4)}=-\frac{1}{k_{1} b_{\left(\frac{S}{C}\right) S 1}^{(1,2,3,4)}}, \quad B_{S 1}^{(1)}=B_{S 1}^{(2)}, B_{S 1}^{(3)}=B_{S 1}^{(4)}
$$

or

$$
b_{\left(\frac{S}{C}\right) S 1}^{(1,2,3,4)}=-\frac{1}{k_{1} B_{S 1}^{(1,2,3,4)}}
$$

$$
l_{\left(\frac{S}{C}\right) S 1}^{(1,2,3,4)}=\frac{\lambda}{2 \pi} \operatorname{atan}\left[b_{\left(\frac{S}{C}\right) S 1}^{(1,2,3,4)}\right]
$$

$\lambda$ is the wavelength.

\subsection{Open circuit (stub)}

$$
B_{S 1}^{(1,2,3,4)}=\frac{b_{\left(\frac{o}{C}\right) s 1}^{(1,2,3,4)}}{k_{1}}, \quad B_{S 1}^{(1)}=B_{S 1}^{(2)}, \quad B_{S 1}^{(3)}=B_{S 1}^{(4)}
$$

or

$$
k_{1} B_{S 1}^{(1,2,3,4)}=b_{\left(\frac{O}{C}\right) S 1}^{(1,2,3,4)}
$$

$$
l_{\left(\frac{O}{C}\right) S 1}^{(1,2,3,4)}=\frac{\lambda}{2 \pi} \operatorname{atan}\left[b_{\left(\frac{O}{C}\right) s 1}^{(1,2,3,4)}\right]
$$


From (41) it can be seen that

$$
B_{1}^{(1)}+B_{1}^{(2)}=\frac{2}{m}
$$

and

$$
\begin{aligned}
& g_{L B 1}=g_{L B 2}=\frac{g_{L A}\left(1+m^{2}\right)}{\left(1-m B_{1}^{(1)}\right)^{2}+m^{2} g_{L A}^{2}} \\
& b_{L B 1}=\frac{m\left(1-g_{L A}^{2}-B_{1}^{(1) 2}\right)+B_{1}^{(1)}\left(1-m^{2}\right)}{\left(1-m B_{1}^{(1)}\right)^{2}+m^{2} g_{L A}^{2}} \\
& b_{L B 2}=\frac{m\left(1-g_{L A}^{2}-B_{1}^{(2) 2}\right)+B_{1}^{(2)}\left(1-m^{2}\right)}{\left(1-m B_{1}^{(1)}\right)^{2}+m^{2} g_{L A}^{2}}
\end{aligned}
$$

Now, we simply find from (10) and (23) the susceptance for the second stub,

$$
B_{S 2}^{(1,2,3,4)}=-b_{L B}^{(1,2)}+\frac{1}{p} \pm g_{L B}^{(1,2)} \sqrt{\frac{g_{(p)}}{g_{L B}^{(1,2)}}-1}
$$

where $g_{L B}^{(1,2)}, b_{L B}^{(1,2)}$ and $g_{(p)}$ are given by (46), (47), (48) and (25).

Now, let us find the corresponding lengths for the second stub:

\subsection{Short circuit (stub)}

$$
B_{S 2}^{(1,2,3,4)}=-\frac{1}{k_{2} n_{\left(\frac{S}{C}\right) S 2}^{(1,2,3,4)}}
$$

or

$$
n_{\left(\frac{S}{C}\right) S 2}^{(1,2,3,4)}=-\frac{1}{k_{2} B_{S 2}^{(1,2,3,4)}}
$$

$l_{\left(\frac{S}{C}\right) S 2}^{(1,2,3)}=\frac{\lambda}{2 \pi} \operatorname{atan}\left[n_{\left(\frac{S}{C}\right) s 2}^{(1,2,3,4)}\right]$

\subsection{Open circuit (stub)}

$$
B_{S 2}^{(1,2,3,4)}=\frac{b_{\left(\frac{O}{C}\right) S 2}^{(1,2,3,4)}}{k_{2}}
$$

or

$$
k_{2} B_{S 2}^{(1,2,3,4)}=n_{\left(\frac{0}{C}\right) s 2}^{(1,2,3,4)}
$$

$$
l_{\left(\frac{o}{C}\right) S 2}^{(1,2,3,4)}=\frac{\lambda}{2 \pi} \operatorname{atan}\left[n_{\left(\frac{O}{C}\right) s 2}^{(1,2,3,4)}\right]
$$

Finally, the susceptance of the third stub can be obtained from (19), 


$$
B_{S 3}^{(1,2,3,4)}=-\frac{p\left(1-g_{L B}^{(1,2) 2}-B_{2}^{(1,2,3,4) 2}\right)+B_{2}^{(1,2,3,4)}\left(1-p^{2}\right)}{\left(1-p B_{2}^{(1,2,3,4)}\right)^{2}+p^{2} g_{L B}^{(1,2) 2}}
$$

where

$g_{L B}^{(1,2)}$ is given by (46).

The corresponding lengths for the third stub are:

\subsection{Short circuit (stub)}

$$
B_{S 3}^{(1,2,3,4)}=-\frac{1}{k_{3} q_{\left(\frac{S}{C}\right) S 3}^{(1,2,3,4)}}
$$

or

$$
q_{\left(\frac{S}{C}\right) S 3}^{(1,2,3,4)}=-\frac{1}{k_{3} B_{S 3}^{(1,2,3,4)}}
$$

$$
l_{\left(\frac{S}{C}\right) S 3}^{(1,2,3,4)}=\frac{\lambda}{2 \pi} \operatorname{atan}\left[q_{\left(\frac{S}{C}\right) s 3}^{(1,2,3,4)}\right]
$$

\subsection{Open circuit (stub)}

$$
B_{S 3}^{(1,2,3,4)}=\frac{q_{\left(\frac{o}{C}\right) S 3}^{(1,2,3,4)}}{k_{3}}
$$

or

$$
k_{3} B_{S 3}^{(1,2,3,4)}=q_{\left(\frac{O}{C}\right) s 3}^{(1,2,3,4)}
$$

$$
l_{\left(\frac{O}{C}\right) S 2}^{(1,2,3,4)}=\frac{\lambda}{2 \pi} \operatorname{atan}\left[q_{\left(\frac{O}{C}\right) s 3}^{(1,2,3,4)}\right]
$$

For stub lengths the following formula must be respected,

$$
l_{s}=\left\{\begin{array}{lc}
\frac{\lambda}{2 \pi} \operatorname{atn}[P]+\frac{\lambda}{2} & \text { for } P<0 \\
\frac{\lambda}{2 \pi} \operatorname{atn}[P] & \text { for } P>0
\end{array}\right\}
$$

In the Table 1. The corresponding solutions are summarized as follows,

Table 1 The stub lengths in the triple stub matching

\begin{tabular}{|l|l|l|}
\hline $\boldsymbol{l}_{\boldsymbol{S 1}}$ & $\boldsymbol{l}_{\boldsymbol{S} \mathbf{2}}$ & $\boldsymbol{l}_{\boldsymbol{S} \mathbf{3}}$ \\
\hline$l_{S 1}^{(1)}$ & $l_{S 2}^{(1)}$ & $l_{S 3}^{(1)}$ \\
\hline$l_{S 1}^{(2)}$ & $l_{S 2}^{(2)}$ & $l_{S 3}^{(2)}$ \\
\hline$l_{S 1}^{(3)}$ & $l_{S 2}^{(3)}$ & $l_{S 3}^{(3)}$ \\
\hline$l_{S 1}^{(4)}$ & $l_{S 2}^{(4)}$ & $l_{S 3}^{(4)}$ \\
\hline
\end{tabular}


This schema is valuable either for the short or the open stubs. Thus, the eight possible combinations can be given by this schema ((S/S/S; S/S/0; 0/S/S; 0/S/0/; S/0/S; S/0/0; 0/0/S; 0/0/0).

In the papers which treat the triple stub matching many authors after finding the lengths of the first stub use it for solving the rest of the problem as the double stub matching. In this paper as it is proposed after finding the length of the first stub, the lengths of the second and the third stubs are simple to find directly from the corresponding formulas. Moreover, in this paper the characteristic impedance of each circuit is different.

2.7. Special cases

a1) $d_{1}=d_{2}=d_{3}=\lambda / 4$

$$
\begin{aligned}
& g_{L A}=\frac{p_{L}}{p_{L}^{2}+q_{L}^{2}}, \quad b_{L A}=-\frac{q_{L}}{p_{L}^{2}+q_{L}^{2}} \\
& g_{L B 1}=g_{L B 2}=\frac{g_{L A}}{B_{1(1)}^{2}+g_{L A}^{2}}=g_{L B} \leq 1 \\
& b_{L B 1}=-\frac{B_{1(1)}}{B_{1(1)}^{2}+g_{L A}^{2}}, \quad b_{L B 2}=-\frac{B_{1(2)}}{B_{1(2)}^{2}+g_{L A}^{2}} \\
& B_{1}^{(1,2)}=b_{L A}+B_{S 1}^{(1,2)} \\
& B_{S 1}^{(1)}=-b_{L A}+g_{L A} \sqrt{Q t-1} \\
& B_{S 1}^{(2)}=-b_{L A}-g_{L A} \sqrt{Q t-1} \\
& Q=\frac{1}{g_{L A}} \\
& B_{S 3}^{(1,2,3,4)}=-\frac{B_{2}^{(1,2,3,4)}}{\left(B_{2}^{(1,2,3,4)}\right)^{2}+g_{L B}^{(1,2) 2}} \\
& B_{S 2}^{(1,2,3,4)}=-b_{L B}^{(1,2)} \pm g_{L B} \sqrt{\frac{1}{g_{L B}}}-1
\end{aligned}
$$

a2) $d_{1}=d_{2}=\lambda / 4, d_{3} \neq \lambda / 4$

$$
\boldsymbol{a}=\boldsymbol{m}=\infty, \quad \boldsymbol{p} \neq \infty
$$

We use (59)-(64)

$$
Q=\frac{g(p)}{g_{L A}}
$$

and (49) and (52) for $B_{S 2}^{(1,2,3,4)}$ and $B_{S 3}^{(1,2,3,4)}$.

a3) $d_{1}=d_{3}=\lambda / 4, d_{2} \neq \lambda / 4$

$$
\boldsymbol{a}=\boldsymbol{p}=\infty, \quad \boldsymbol{m} \neq \infty
$$

We use (56) for $g_{L A}, b_{L A},(46)$, (48 and (58) for $g_{L B 1}, g_{L B 2}, b_{L B 1}, b_{L B 2}$, (3) (40) and (41) for $B_{1}^{(1,2)}, B_{S 1}^{(1,2)}$. For $B_{S 2}^{(1,2,3,4)}$ and $B_{S 3}^{(1,2,3,4)}(63)$ and (64) are used respectively with, 


$$
Q=\frac{g(m)}{g_{L B}}
$$

a4) $d_{1}=\lambda / 4, d_{2} \neq \lambda / 4 \quad d_{3} \neq \lambda / 4$

$$
\boldsymbol{a}=\infty, \quad \boldsymbol{m} \neq \infty, \quad \boldsymbol{p} \neq \infty
$$

We use (59) for $g_{L A}, b_{L A}$. All other expressions for $B_{S 1}^{(1,2)}, B_{S 2}^{(1,2,3,4)}$ and $B_{S 3}^{(1,2,3,4)}$ are calculated by the same expressions as in the general case.

a5) $d_{1} \neq \frac{\lambda}{4}, d_{2}=\lambda / 4 \quad d_{3} \neq \lambda / 4$

$$
\boldsymbol{a} \neq \infty, \quad \boldsymbol{m}=\infty, \quad \boldsymbol{p} \neq \infty
$$

We use (60) and (61) for calculating $B_{S 1}^{(1,2)},(57)$ and (58) for $g_{L B}, b_{L B 1}, b_{L B 2}$ with

$$
Q=\frac{1}{g(p) g_{L B}}-1
$$

All other expressions for $B_{S 2}^{(1,2,3,4)}$ and $B_{S 3}^{(1,2,3,4)}$ are calculated by the same expressions as in the general case.

a6) $d_{1} \neq \frac{\lambda}{4}, d_{2} \neq \frac{\lambda}{4} d_{3}=\lambda / 4$

$$
\boldsymbol{a} \neq \infty, \quad \boldsymbol{m} \neq \infty, \quad \boldsymbol{p}=\infty
$$

We use (63) and (64) for calculating $B_{S 2}^{(1,2,3,4)}$ and $B_{S 3}^{(1,2,3,4)}$ with (66). All other expressions are calculated by the same expressions as in the general case.

The stub lengths either for short circuit or open circuit are calculated by the previous expressions

\section{Numerical Validation}

To verify the validity of the approved analytical method, some problems solved by the Smith chart will be treated.

3.1. The normalized terminating impendence is $z_{L N}=(1.64+j 1.97) \Omega$ and the normalized characteristic impendences of the line and the stubs are $1 \Omega$. The first stub is away $d_{1}=0.154 \lambda$ from the load. The spacing between the first and second stub is stub $d_{2}=1 / 8 \lambda$ and between the third and the second stub is $d_{3}=1 / 8 \lambda$.

3.1.1. Determine the lengths of the short-circuited stubs as well as the open circuited stubs when the match is achieved.

\subsubsection{Find the SWR on any section of the transmission line.}

Solution:

Short stubs:

In [2] the incomplete solution of this problem is given for the lengths of three stubs.

Using the Smith chart [2] gives,

First stub:

$$
l_{1}^{(1)}=0.367 \lambda \text { and } l_{1}^{(2)}=0.203 \lambda
$$

Second stub: 


$$
l_{2}^{(1)}=0.312 \lambda, l_{2}^{(2)}=0.471 \lambda, l_{2}^{(3)}=0.100 \lambda, l_{2}^{(4)}=0.457 \lambda
$$

Third stub:

$$
l_{3}^{(1)}=0.367 \lambda \text { and } l_{3}^{(2)}=0.203 \lambda
$$

From the presented method:

$$
\begin{gathered}
a=\tan \left(\beta d_{1}\right)=1.45175 \\
m=\tan \left(\beta d_{2}\right)=1, \quad p=\tan \left(\beta d_{3}\right)=1 \\
g_{L A}=0.3540, \quad b_{L A}=0.7132
\end{gathered}
$$

$Q=2.8251, t=0.3540<1$, There is not the unique solution.

Let us begin with $t=1$, (38) for which the minimum limits of the first stub are given by (36) and (37),

$$
B_{S 1}^{(1)}>-b_{L A}+\frac{1}{m}+g_{L A} \sqrt{Q-1}=0.7650
$$

and

$$
B_{S 1}^{(1)}<-b_{L A}+\frac{1}{m}-g_{L A} \sqrt{Q-1}=-0.1914
$$

Thus,

$$
\begin{gathered}
B_{S 1}^{(1)}>0.7650 \\
B_{S 1}^{(2)}<-0.1914
\end{gathered}
$$

are the limits for the first stub or more accurately the domain in which the matching is possible.

Just for these limits we obtained (the case $t=1$ ),

$$
\begin{gathered}
l_{S 1}^{(1)}=l_{S 1}^{(2)}=0.3539 \lambda \text { and } l_{S 1}^{(3)}=l_{S 1}^{(4)}=0.2199 \lambda \\
l_{S 2}^{(1)}=l_{S 2}^{(2)}=0.4666 \lambda \text { and } l_{S 2}^{(3)}=l_{S 2}^{(4)}=0.1526 \lambda \\
l_{S 3}^{(1)}=l_{S 3}^{(2)}=l_{S 3}^{(3)}=l_{S 3}^{(4)}=0.3750 \lambda
\end{gathered}
$$

The same results can be obtained by [1]. In [2] using the Smith chart the susceptance for the first stub is obtained as follows,

$$
B_{S 1(1)}=0.9 \text { and } B_{S 1(1)}=-0.3
$$

from which the lengths of the first stub are previously given.

The limits for the first stub are (36),

$$
B_{S 1}^{(1)}>0.7650 \text { and } B_{S 1}^{(1)}<-0.1914
$$

Obviously, these values are in the domain of definition for the matching,

$$
0.9>0.7650
$$


and

$$
-0.3<-0.1914
$$

Possibly in [2] these values were chosen randomly to be sure that the load will be outside of the forbidden region.

By inspection and with [12] we find $t=1.37931$ which could correspond to the results obtained by the previous values for the susceptance of the first stub, [2].

By [12] the susceptances for the first stub are,

$$
B_{S 1}^{(1)}=0.8892, \quad B_{S 1}^{(2)}=-0.3157
$$

These values satisfy (36) and (37).

The limits for the second stub (28) are,

$$
3.4679 \leq B_{S 2}^{(1,2)} \leq 5.4679
$$

and

$$
-1.4679 \leq B_{S 2}^{(3.4)} \leq 0.5321
$$

For the second stab the calculate values are,

$B_{S 2}^{(1)}=5.3609, \quad B_{S 2}^{(2)}=3.5748$

$B_{S 2}^{(3)}=0.4252, \quad B_{S 2}^{(4)}=-1.3609$

Obviously, all susceptances for the second stub belong to previously domain of the matching.

The lengths of each stub which can be summarized in the Table 2.

Table 2 The stub lengths $(\mathrm{S} / \mathrm{C})$

\begin{tabular}{|c|c|c|}
\hline $\mathbf{l}_{\mathbf{S} 1} / \boldsymbol{\lambda}$ & $\mathbf{l}_{\mathbf{S} 2} / \boldsymbol{\lambda}$ & $\mathbf{l}_{\mathbf{S} 3} / \boldsymbol{\lambda}$ \\
\hline 0.3657 & 0.4566 & 0.3084 \\
\hline 0.3657 & 0.4706 & 0.4118 \\
\hline 0.2014 & 0.1008 & 0.3084 \\
\hline 0.2014 & 0.3141 & 0.4118 \\
\hline
\end{tabular}

The obtained results correspond to those for the first and the second stub obtained by the Smith chart [2] but not for the third stub.

By using [1] we obtained the same results as with our analytical approach

Let us prove the validity of the results obtained analytically for the third row.

We use the standing wave ratio SWR, [11].

I) SWR before the load:

$$
d_{1 z N}=0, \quad l_{1 z N}=0
$$




$$
\begin{gathered}
z_{1 N}=(1.64+\mathrm{j} j 1.79) \Omega \\
\left|\Gamma_{\text {BLoad }}\right|=0.6288, \quad S W R_{\text {BLoad }}=4.3883
\end{gathered}
$$

II)SWR between the first and the second stub:

$$
\begin{gathered}
d_{1}=0.154 \lambda, \quad l_{S 1}^{(1)}=0.2014 \lambda \\
z_{\text {Totals } 1}=(1.64+j 1.79) \Omega \\
\left|\Gamma_{(S 1-S 2)}\right|=0.5375, \quad S W R_{(S 1-S 2)}=3.3242
\end{gathered}
$$

III) SWR between the second and third stub:

$$
\begin{gathered}
z_{\text {Totals } 2}=(1.2501-j 1.4032) \Omega \\
d_{2}=0.125 \lambda, \quad l_{S 2}^{(1)}=0.1008 \lambda \\
\left|\Gamma_{(S 2-S 3)}\right|=0.1884, \quad S W R_{(S 2-S 3)}=1.4641
\end{gathered}
$$

IV) SWR before the third stub:

$$
\begin{gathered}
z_{T S 3}=(0.6863-j 0.0508) \Omega \\
d_{3}=0.125 \lambda, \quad l_{S 3}^{(1)}=0.3084 \lambda \\
\left|\Gamma_{(B S 3)}\right|=3.9793 \cdot 10^{-4}, S W R_{(B S 3)}=1.0008
\end{gathered}
$$

Thus, the calculations are proved. In these calculations $\Gamma$ and $S W R$ are the reflection coefficient and the standing wave ratio, respectively.

The potential users can verify the reflection coefficient and the standing wave ratio for other solutions of the stub lengths.

Open stubs:

Table 3 The stub lengths $(\mathrm{O} / \mathrm{C})$

\begin{tabular}{|c|c|c|}
\hline $\boldsymbol{l}_{\boldsymbol{S} 1} / \boldsymbol{\lambda}$ & $\boldsymbol{l}_{\boldsymbol{S} 2} / \boldsymbol{\lambda}$ & $\boldsymbol{l}_{\boldsymbol{S} 3} / \boldsymbol{\lambda}$ \\
\hline 0.1157 & 0.2206 & 0.1618 \\
\hline 0.1157 & 0.2066 & 0.0584 \\
\hline 0.4513 & 0.0640 & 0.1618 \\
\hline 0.4513 & 0.3509 & 0.0584 \\
\hline
\end{tabular}

By using [1] we obtained the same results.

3.2. The terminating impendence is $Z_{L}=(50-j 10) \Omega$ and the characteristic impendence $Z_{0}$ of the line and the stubs is $50 \Omega$. The first stub is connected to the load $\left(d_{1}=0 \lambda\right)$. The spacing between the first and second stub is stub $d_{2}=1 / 8 \lambda$ and between the third and the second stub is $d_{3}=1 / 8 \lambda$. Determine the lengths of the shortcircuited stubs as well as the open circuited stubs when the match is achieved.

Solution:

Short stubs:

From the presented method:

$$
\begin{gathered}
\mathrm{a}=\tan \left(\beta d_{1}\right)=0 \\
m=\tan \left(\beta d_{2}\right)=1, \quad p=\tan \left(\beta d_{3}\right)=1
\end{gathered}
$$




$$
g_{L A}=0.9615, \quad b_{L A}=0.1923
$$

$Q=1.0400, t=0.9615<1$, There is not the unique solution.

$$
\begin{gathered}
g_{L B 1}=g_{L B 2}=1.6 \\
b_{L B 1}=-1.8764, \quad b_{L B 2}=-0.1236 \\
B_{S 1}^{(1)}=1.3343, \quad B_{S 1}^{(2)}=0.281
\end{gathered}
$$

The limits for the first stub are (36),

$$
\begin{aligned}
& B_{S 1}^{(1)} \geq 1.3342 \\
& B_{S 1}^{(2)} \leq 0.2812
\end{aligned}
$$

The limits for the second stub (28) are,

$$
\begin{gathered}
B_{S 2}^{(1)}=3.6764, \quad B_{S 2}^{(2)}=2.0764 \\
B_{S 3}^{(3)}=1.9236, \quad B_{S 2}^{(4)}=0.3236 \\
1.8764 \leq B_{S 2}^{(1,2)} \leq 3.8764
\end{gathered}
$$

and

$$
0.1236 \leq B_{S 2}^{(3.4)} \leq 2.1236
$$

Well, all susceptance for the first and second stub are in the domain of the matching.

Fort $=1.25$, from this work and (11) the results are given in Tables 4 and 5 .

Table 4 The stub lengths in the triple stub matching (S/C)

\begin{tabular}{|l|l|l|}
\hline $\boldsymbol{l}_{\boldsymbol{S} 1} / \boldsymbol{\lambda}$ & $\boldsymbol{l}_{\boldsymbol{S} 2} / \boldsymbol{\lambda}$ & $\boldsymbol{l}_{\boldsymbol{S} 3} / \boldsymbol{\lambda}$ \\
\hline 0.3976 & 0.4577 & 0.4064 \\
\hline 0.3976 & 0.4286 & 0.3238 \\
\hline 0.2936 & 0.4237 & 0.4064 \\
\hline 0.2936 & 0.2998 & 0.3238 \\
\hline
\end{tabular}

Open stubs:

Table 5 The stub lengths $(\mathrm{O} / \mathrm{C})$

\begin{tabular}{|c|c|c|}
\hline $\boldsymbol{l}_{\boldsymbol{s} 1} / \boldsymbol{\lambda}$ & $\boldsymbol{l}_{\boldsymbol{s} 2} / \boldsymbol{\lambda}$ & $\boldsymbol{l}_{\boldsymbol{S 3}} / \boldsymbol{\lambda}$ \\
\hline 0.1476 & 0.2077 & 0.1564 \\
\hline 0.1476 & 0.1786 & 0.0738 \\
\hline 0.0436 & 0.1737 & 0.1564 \\
\hline 0.0436 & 0.0498 & 0.0738 \\
\hline
\end{tabular}

Using [1] we obtain the same results. 
3.3. The terminating impendence is $Z_{L}=(100+j 50) \Omega$ and the characteristic impendence $Z_{0}$ of the line and the stubs is $50 \Omega$. The first stub is away $d_{1}=0.503 \lambda$ from the load. The spacing between the first and second stub is stub $d_{2}=3 / 8 \lambda$ and between the third and the second stub is $d_{3}=3 / 8 \lambda$. Determine the lengths of the short-circuited stubs as well as of the open circuited stubs when the match is achieved.

Solution:

$$
\begin{gathered}
\mathrm{a}=\tan \left(\beta d_{1}\right)=0.0189 \\
m=\tan \left(\beta d_{2}\right)=-1, \quad p=\tan \left(\beta d_{3}\right)=-1
\end{gathered}
$$

$Q=1.0400, t=0.9615<1$, There is not the unique solution.

$$
\begin{gathered}
g_{L A}=0.3971, \quad b_{L A}=0.1923 \\
g_{L B 1}=g_{L B 2}=1.6 \\
b_{L B 1}=-1.8764, \quad b_{L B 2}=-0.1236
\end{gathered}
$$

Short stubs: (for $t=1.75$ )

Table 6 The stub lengths (S/C)

\begin{tabular}{|l|l|l|}
\hline $\boldsymbol{l}_{\boldsymbol{s} \mathbf{1}} / \boldsymbol{\lambda}$ & $\boldsymbol{l}_{\boldsymbol{s} \mathbf{2}} / \boldsymbol{\lambda}$ & $\boldsymbol{l}_{\boldsymbol{s} \mathbf{3}} / \boldsymbol{\lambda}$ \\
\hline 0.2367 & 0.3825 & 0.2288 \\
\hline 0.2367 & 0.1351 & 0.0783 \\
\hline 0.0912 & 0.0494 & 0.2288 \\
\hline 0.0912 & 0.0308 & 0.0783 \\
\hline
\end{tabular}

Open stubs: (for $t=1.75$ )

Table 7 The stub lengths $(\mathrm{O} / \mathrm{C})$

\begin{tabular}{|l|l|l|}
\hline $\boldsymbol{l}_{\boldsymbol{S} \mathbf{1}} / \boldsymbol{\lambda}$ & $\boldsymbol{l}_{\boldsymbol{S} 2} / \boldsymbol{\lambda}$ & $\boldsymbol{l}_{\boldsymbol{S 3}} / \boldsymbol{\lambda}$ \\
\hline 0.4867 & 0.1325 & 0.4788 \\
\hline 0.4867 & 0.3851 & 0.3283 \\
\hline 0.3412 & 0.2994 & 0.4788 \\
\hline 0.3412 & 0.2808 & 0.3283 \\
\hline
\end{tabular}

Using [1] we obtain the same results.

3.4. Let us solve the following problem where the terminating impendence is $Z_{L}=(25-j 25) \Omega$ and the characteristic impendence $Z_{0}$ of the line and the stubs is $50 \Omega$. The first stub is away $\mathrm{d}_{1}=0.25 \lambda$ from the load. The spacing between the first and second stub is stub $\mathrm{d}_{2}=1 / 4 \lambda$ and between the third and the second stub is $d_{3}=1 / 4 \lambda$. Determine the lengths of the short-circuited stubs as well as of the open circuited stubs when the match is achieved.

Solution:

This is the special case a1) 
Table 8 The stub lengths (S/C): For $t=10$

\begin{tabular}{|c|c|c|}
\hline $\boldsymbol{l}_{\boldsymbol{s} \mathbf{1}} / \boldsymbol{\lambda}$ & $\boldsymbol{l}_{\boldsymbol{S} 2} / \boldsymbol{\lambda}$ & $\boldsymbol{l}_{\boldsymbol{S} 3} / \boldsymbol{\lambda}$ \\
\hline 0.4431 & 0.3510 & 0.4488 \\
\hline 0.4431 & 0.2715 & 0.0512 \\
\hline 0.0855 & 0.285 & 0.4488 \\
\hline 0.0855 & 0.1490 & 0.0512 \\
\hline
\end{tabular}

Table 9 The stub lengths (O/C): For $t=10$

\begin{tabular}{|l|l|l|}
\hline $\boldsymbol{l}_{\boldsymbol{s} \mathbf{1}} / \boldsymbol{\lambda}$ & $\boldsymbol{l}_{\boldsymbol{s} \mathbf{2}} / \boldsymbol{\lambda}$ & $\boldsymbol{l}_{\boldsymbol{s} \mathbf{3}} / \boldsymbol{\lambda}$ \\
\hline 0.1931 & 0.1010 & 0.1988 \\
\hline 0.1931 & 0.0215 & 0.3012 \\
\hline 0.3355 & 0.4785 & 0.1988 \\
\hline 0.3355 & 0.3990 & 0.3012 \\
\hline
\end{tabular}

Using [1] we obtain the same results either for the short stubs or for the open stubs.

3.5. A load with a load impedance of $Z_{L}=(10+j 5) \Omega$ is to be matched to a transmission line of a microstrip line with a characteristic input impedance of $50 \Omega$ using a triple stub. All stubs are open with the same characteristic impedances as the transmission line. The gaps between the stubs are $d_{2}=3 / 8 \lambda$ and $d_{3}=3 / 8 \lambda$, and the gap between the load and the first stub is fixed at $d_{1}=0.503 \lambda$.

Solution:

In this case,

$$
\begin{gathered}
\mathrm{a}=\tan \left(\beta d_{1}\right)=0.0189 \\
m=\tan \left(\beta d_{2}\right)=-1, \quad p=\tan \left(\beta d_{3}\right)=-1 \\
g_{L A}=3.6964>1, \quad b_{L A}=-2.1778 \\
Q=0.2705<1, \quad t=3.6959
\end{gathered}
$$

This is the case of the unique solution where all expressions do not depend on the parameter ' $t$ ', (37) and (38) for,

$$
\begin{gathered}
1 \leq t \leq t_{\text {min }}=3.6969 \\
g_{L B 1}=g_{L B 2}=0.5411 \\
b_{L B 1}=b_{L B 2}=1 \\
B_{S 1}^{(1)}=B_{S 1}^{(2)}=1.1778 \\
B_{S 2}^{(1)}=B_{S 2}^{(3)}=-1.1115, \quad B_{S 2}^{(2)}=B_{S 2}^{(4)}=-2.8885 \\
B_{S 3}^{(1)}=B_{S 3}^{(3)}=0.6421, \quad B_{S 23}^{(2)}=B_{S 3}^{(4)}=-2.6421
\end{gathered}
$$

For any ' $t$ ' from the interval $1 \leq t \leq t_{\min }=3.6969$ the unique solution is given for the open and the short stubs in Tables 10 and 11, successively. Let us take $t=3$ from this interval where we have the unique solution. 
Table 10 The stub lengths $(\mathrm{O} / \mathrm{C})$

\begin{tabular}{|l|l|l|}
\hline $\boldsymbol{l}_{\boldsymbol{S} \mathbf{1}} / \boldsymbol{\lambda}$ & $\boldsymbol{l}_{\boldsymbol{S} \mathbf{2}} / \boldsymbol{\lambda}$ & $\boldsymbol{l}_{\boldsymbol{S 3}} / \boldsymbol{\lambda}$ \\
\hline 0.1380 & 0.3666 & 0.0908 \\
\hline 0.1380 & 0.3030 & 0.3076 \\
\hline 0.1380 & 0.3666 & 0.0908 \\
\hline 0.1380 & 0.3030 & 0.3076 \\
\hline
\end{tabular}

Table 11 The stub lengths (S/C)

\begin{tabular}{|l|l|l|}
\hline $\boldsymbol{l}_{\boldsymbol{S 1}} / \boldsymbol{\lambda}$ & $\boldsymbol{l}_{\boldsymbol{S 2}} / \boldsymbol{\lambda}$ & $\boldsymbol{l}_{\boldsymbol{S 3}} / \boldsymbol{\lambda}$ \\
\hline 0.3880 & 0.1166 & 0.3408 \\
\hline 0.3880 & 0.0530 & 0.0576 \\
\hline 0.3880 & 0.1166 & 0.3408 \\
\hline 0.3880 & 0.0530 & 0.0576 \\
\hline
\end{tabular}

By [1] the same results are obtained.

For any ${ }^{\prime} t^{\prime}$ from the interval $t=t_{\min }>3.6964$ we obtain the different solutions as it has been approved previously. Many tests have been made and confirmed by [1].

3.6. A load with a load impedance of $Z_{L}=(50-j 150) \Omega$ is to be matched to a transmission line of a microstrip line with a characteristic input impedance of $50 \Omega$ using a triple stub. All stubs are open with the same characteristic impedances as the transmission line. The gaps between the stubs are $\mathrm{d}_{2}=1 / 8 \lambda$ and $\mathrm{d}_{3}=3 / 8 \lambda$, and the gap between the load and the first stub is fixed at $\mathrm{d}_{1}=0.2 \lambda$.

$$
\begin{gathered}
\mathrm{a}=\tan \left(\beta d_{1}\right)=3.0777 \\
m=\tan \left(\beta d_{2}\right)=1, \quad p=\tan \left(\beta d_{3}\right)=-1 \\
g_{L A}=10.4093>1, \quad b_{L A}=2.2691 \\
Q=0.0961<1, \quad t=10.4058
\end{gathered}
$$

This is the case of the unique solution where all expressions do not depend on the parameter' $t^{\prime},(37)$ and (38) for,

$$
1 \leq t \leq t_{\min }=10.4058
$$

For any' $t$ ' from the interval $1 \leq t \leq t_{\min }=10.4058$ the unique solution is given for the open and the short stub in Tables 12 and 3 , successively. Let us take $t=7$ from this interval where we have the unique solution.

Table 12 The stub lengths $(\mathrm{O} / \mathrm{C})$

\begin{tabular}{|l|l|l|}
\hline $\boldsymbol{l}_{\boldsymbol{S} \mathbf{1}} / \boldsymbol{\lambda}$ & $\boldsymbol{l}_{\boldsymbol{S} \mathbf{2}} / \boldsymbol{\lambda}$ & $\boldsymbol{l}_{\boldsymbol{S} \mathbf{3}} / \boldsymbol{\lambda}$ \\
\hline 0.3562 & 0.0848 & 0.1783 \\
\hline 0.3562 & 0.4152 & 0.2884 \\
\hline 0.3562 & 0.0848 & 0.1783 \\
\hline 0.3562 & 0.4152 & 0.2884 \\
\hline
\end{tabular}


Table 13 The stub lengths (S/C)

\begin{tabular}{|l|l|l|}
\hline $\boldsymbol{l}_{\boldsymbol{s} 1} / \boldsymbol{\lambda}$ & $\boldsymbol{l}_{\boldsymbol{s} 2} / \boldsymbol{\lambda}$ & $\boldsymbol{l}_{\boldsymbol{s} 3} / \boldsymbol{\lambda}$ \\
\hline 0.1062 & 0.3348 & 0.4283 \\
\hline 0.1062 & 0.1652 & 0.0384 \\
\hline 0.1062 & 0.3348 & 0.4283 \\
\hline 0.1062 & 0.1652 & 0.0384 \\
\hline
\end{tabular}

By [1] the same results are obtained. One can see that in the large diapason of the change of ' $t$ ' where the unique triple stub matching is achieved.

For any ' $t$ ' from the interval $t=t_{\min }>10.4058$ we obtain the different solutions as it has been approved previously. Many tests have been made and confirmed by [1].

3.7. Finally, let us solve the following example in which all characteristic impedances are different. This example can be used to verify the triple stub matching by using the Smith chart. All characteristic impedances are different.

The terminating impendence is $Z_{L}=(100+j 100) \Omega$ and the characteristic impendence of the line $Z_{0}$ is $50 \Omega$. The characteristic impedances of stubs are respectively $Z_{S 1}=75 \Omega, Z_{S 2}=100 \Omega$ and $Z_{S 3}=125 \Omega$. The first stub is away $\mathrm{d}_{1}=$ $0.4 \lambda$ from the load. The spacing between the first and second stub is stub $d_{2}=3 / 8 \lambda$ and between the third and the second stub is $d_{3}=1 / 8 \lambda$. Determine the lengths of the open stubs as well as the short-circuited stubs when the match is achieved.

Solution:

Open stubs:

We start with the extreme case, that is, with $x=1$ for which we have,

$$
\begin{gathered}
\mathrm{a}=\tan \left(\beta d_{1}\right)=-0.7265 \\
m=\tan \left(\beta d_{2}\right)=-1, \quad p=\tan \left(\beta d_{3}\right)=1 \\
g_{L A}=0.5436, \quad b_{L A}=-1.0726
\end{gathered}
$$

$Q=1.8397, t=0.5436<1$, There is not the unique solution.

$$
\begin{gathered}
g_{L B 1}=g_{L B 2}=2 \\
b_{L B 1}=-0.8327, \quad b_{L B 2}=2.8327 \\
B_{S 1}^{(1)}=0.5707, \quad B_{S 1}^{(2)}=-0.4255 \\
B_{S 1}^{(1)} \geq 0.5707 \\
B_{S 1}^{(2)} \leq-0.4255
\end{gathered}
$$

It was expected because $B_{S 1}^{(1)}$ and $B_{S 1}^{(2)}$ are the limits of the domain of the matching.

$$
\begin{gathered}
B_{S 2}^{(1)}=1.8327, \quad B_{S 2}^{(2)}=1.8327 \\
B_{S 3}^{(3)}=-1.8327, B_{S 2}^{(4)}=-1.8327 \\
1.8327 \leq B_{S 2}^{(1,2)} \leq 3.8327
\end{gathered}
$$


and

$$
-2.8327 \leq B_{S 2}^{(3.4)} \leq-1.8327
$$

The same conclusion is for $B_{S 1}^{(1,2)}$ and $B_{S 3}^{(3,4)}$ which are the limits of the domain of matching. The stub lengths are summarized in Table 14.

Table 14 The stub lengths $(\mathrm{O} / \mathrm{C})$

\begin{tabular}{|l|l|l|}
\hline $\boldsymbol{l}_{\boldsymbol{S} 1} / \boldsymbol{\lambda}$ & $\boldsymbol{l}_{\boldsymbol{S} 2} / \boldsymbol{\lambda}$ & $\boldsymbol{l}_{\boldsymbol{S} 3} / \boldsymbol{\lambda}$ \\
\hline 0.1127 & 0.2076 & 0.1894 \\
\hline 0.1127 & 0.2076 & 0.1894 \\
\hline 0.4096 & 0.2924 & 0.1894 \\
\hline 0.4096 & 0.2924 & 0.1894 \\
\hline
\end{tabular}

Short stubs:

In Table 15 are summarized the lengths of short stubs.

Table 15 The stub lengths (S/C)

\begin{tabular}{|l|l|l|}
\hline $\boldsymbol{l}_{\boldsymbol{S 1}} / \boldsymbol{\lambda}$ & $\boldsymbol{l}_{\boldsymbol{S 2}} / \boldsymbol{\lambda}$ & $\boldsymbol{l}_{\boldsymbol{S 3}} / \boldsymbol{\lambda}$ \\
\hline 0.3627 & 0.4576 & 0.4394 \\
\hline 0.3627 & 0.4576 & 0.4394 \\
\hline 0.1596 & 0.0424 & 0.4394 \\
\hline 0.1596 & 0.0424 & 0.4394 \\
\hline
\end{tabular}

Now, let us solve the same example for $x=1.5$.

In Table 16 and Table 17 are summarized the lengths for the open and short stubs, respectively.

Open stubs:

Table 16 The stub lengths (O/C)

\begin{tabular}{|c|c|c|}
\hline $\boldsymbol{l}_{\boldsymbol{S 1}} / \boldsymbol{\lambda}$ & $\boldsymbol{l}_{\boldsymbol{s} 2} / \boldsymbol{\lambda}$ & $\boldsymbol{l}_{\boldsymbol{S} 3} / \boldsymbol{\lambda}$ \\
\hline 0.1388 & 0.2210 & 0.2134 \\
\hline 0.1388 & 0.1634 & 0.1006 \\
\hline 0.3772 & 0.3366 & 0.2134 \\
\hline 0.3772 & 0.2790 & 0.1006 \\
\hline
\end{tabular}


Short stubs:

Table 17 The stub lengths $(\mathrm{S} / \mathrm{C})$

\begin{tabular}{|c|c|c|}
\hline $\boldsymbol{l}_{\boldsymbol{s} 1} / \boldsymbol{\lambda}$ & $\boldsymbol{l}_{\boldsymbol{s} 2} / \boldsymbol{\lambda}$ & $\boldsymbol{l}_{\boldsymbol{s} 3} / \boldsymbol{\lambda}$ \\
\hline 0.3888 & 0.4710 & 0.4634 \\
\hline 0.3888 & 0.4134 & 0.3506 \\
\hline 0.1272 & 0.0866 & 0.4634 \\
\hline 0.1272 & 0.0290 & 0.3506 \\
\hline
\end{tabular}

Again, we use the standing wave ratio SWR, [11].

The stub lengths are from the fourth row (Table 17, S/C):

$$
l_{S 1}=0.1272 \lambda, \quad l_{S 2}=0.0290 \lambda, \quad l_{S 3}=0.3506 \lambda
$$

I) SWR before the load:

$$
\begin{gathered}
d_{1 z N}=0, \quad l_{1 z N}=0 \\
z_{1 N}=(2+j 2) \Omega \\
\left|\Gamma_{\text {BLoad }}\right|=0.6202, \quad V S W R_{\text {BLoad }}=4.2656
\end{gathered}
$$

II)SWR between the first and the second stub:

$$
\begin{gathered}
d_{1}=0.4 \lambda, \quad l_{S 1}^{(1)}=0.1272 \lambda \\
Z_{\text {Total } 1}=(8.3426+j 26.4160) \Omega \\
\left|\Gamma_{(S 1-S 2)}\right|=0.7702, \quad V S W R_{(S 1-S 2)}=7.7033
\end{gathered}
$$

III) SWR between the second and third stub:

$$
\begin{gathered}
z_{\text {Totals } 2}=(37.4429-j 1.5441) \Omega \\
d_{2}=0.375 \lambda, \quad l_{S 2}^{(1)}=0.0290 \lambda \\
\left|\Gamma_{(S 2-S 3)}\right|=0.1447, \quad V S W R_{(S 2-S 3)}=1.3383
\end{gathered}
$$

IV) SWR before the third stub:

$$
\begin{gathered}
z_{T S 3}=(50.0755-j 0.0377) \Omega \\
d_{3}=0.125 \lambda, \quad l_{S 3}^{(1)}=0.3506 \lambda \\
\left|\Gamma_{(B S 3)}\right|=8.4298 \cdot 10^{-4} \\
\operatorname{VSWR}_{(B S 3)}=1.0017
\end{gathered}
$$

Thus, the calculations are proved. In these calculations $\Gamma$ and $S W R$ are the reflection coefficient and the standing wave ratio, respectively. 


\section{Conclusion}

In this paper we present the improved analytical approach for the triple stub matching. The lengths of all stubs were found as well as the domains of the matching for the first and second stubs. Even though there are many possibilities of the matching the previous domains must be respected. Moreover, in this paper all characteristic impedances either for the line or for the stubs are different. The paper is written in detail with the general and the special cases. Many representative examples are solved in detail so that potential readers can easily follow and understand them. It is recommended to verify these examples by using the Smith chart. In this paper we treated the short and open stubs bath other their combinations are possible. We propose this paper as the educational tool for the students and people which work in this domain because they can make the own MATLAB or MATHEMATICA codes using the formulas given in this work.

\section{Compliance with ethical standards}

\section{Disclosure of conflict of interest}

Authors declare no conflict of interest.

\section{References}

[1] Orfanidis SJ. Electromagnetic Waves and Antennas, Rutgers University.

[2] Townsend. AAA. The Smith chart and its Applications, Ingeneria/TEM\%20//Material\%29Vario/Smith_Book_Complete.pdf.

[3] Pozar DM. 'Microwave Engineering', Fourth Edition.

[4] Jackson JD. Classical Electrodynamics, John Wiley and Sons Inc., Third Edition, New York. 1998.

[5] Benson FA, Benson TM. Fields, Waves and Transmission lines, Springer-Science \& Business Media, BV.

[6] Davidson SB. Computational Electromagnetics for RF and Microwave Engineering, 2nd Edition. November 2010.

[7] Collin RE. Foundations for Microwave Engineering, 2nd Edition. January 2001.

[8] Kraus JD. Electromagnetics, Third edition McGraw-Hill Book Company.

[9] Collier R. Transmission lines, 1st Edition, ISBN-10: 1107026008.

[10] Balanis CA. 'Engineering Electromagnetics', ISBN: 0471621943.

[11] Babic S, Akyel C. An improved analytical method for triple stub matching (stubs in series), Global Journal of Engineering and Technology Advances, 2021, 07 (02), 103-123.

[12] Babic S, Akyel C. Software- Matching (MATLAB). 2019. 